\title{
In situ Imaging of Single Polyelectrolyte Chains with the Atomic Force Microscope
}

\author{
Svilen Kozhuharov, Plinio Maroni, and Michal Borkovec*
}

\begin{abstract}
This article discusses the possibilities offered by modern atomic force microscopes (AFMs) with ultrasmall cantilevers to perform in situ imaging of single adsorbed polyelectrolytes in aqueous solutions. We demonstrate that such AFM techniques permit high quality images of single polyelectrolyte molecules to be obtained. These images can then be used to qualitatively address differences in the adsorbed conformations for different polyelectrolyte architectures. Moreover, such images can be also analyzed quantitatively. As an example, we discuss the determination of the persistence length of adsorbed polyelectrolytes.
\end{abstract}

Keywords: Adsorption · AFM · Conformation · Image analysis · Mica · Polyelectrolyte

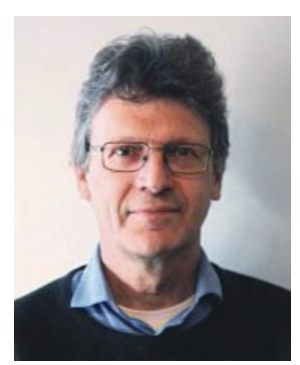

Prof. Michal Borkovec studied chemistry at the University of Bern, Switzerland, and got his PhD in 1986 at Columbia University, New York City. Thereafter he worked at the University of Basel, and then as a lecturer at the Swiss Federal Institute of Technology in Zürich. He was appointed as a professor in 1998 at Clarkson University, Postdam, New York. In 2001, he moved to the University of Geneva, where he continues to work until now. His main research focus is physical and analytical chemistry of colloids, surfaces, and polymers. His recent activities are direct force measurements and investigations of single molecules. $\mathrm{He}$ also worked in the Swiss National Research Council, and is currently a member of the Swiss Academy of Engineering Sciences.

\section{Introduction}

The dream of many chemists is to perform analysis on a single molecule level. Only rather recently, this idea started to become reality. One line of exciting research is to use sensitive optical detection techniques and collect fluorescence signals from single molecules. ${ }^{[1,2]}$ The other line is to use scanning probe techniques, especially the atomic force microscope (AFM). ${ }^{[3]}$ These techniques allow the investigation of surfaces down to single atoms and molecules. A particular challenge in the context of biologically relevant materials is to perform this kind of analysis in aqueous media. One is met with the same challenge for many bio-inspired engineered materials. Besides generating reliable images, the AFM also permits the measurements of the stretching of individual polymer chains, studies of conformational transitions, or of the analysis of individual desorption events. ${ }^{[4-7]}$

This short article will focus on the second line, and we will particularly demonstrate the capabilities of a modern AFM to image adsorbed single polyelectrolyte molecules on solid substrates in aqueous environments. While this goal has been achieved under dry conditions in the recent past, ${ }^{[8]}$ current instrumentation now permits a similar type of analysis in aqueous solutions. To reliably image such systems in situ represents a prerequisite for single molecule analysis and manipulation of biologically relevant systems. More details on similar experiments can be found in a recent publication. ${ }^{[9]}$

\section{Atomic Force Microscope (AFM)}

Binnig and coworkers ${ }^{[3]}$ described the AFM in 1986, which was realized in the IBM laboratories in Rüschlikon, Switzerland. This instrument represented a variant of the scanning tunneling microscope (STM) developed earlier, for which the Nobel Prize was awarded in the same year. In the meantime, however, the AFM has become an extremely popular research tool, more so than the STM.

The scheme of an AFM is shown in Fig. 1. In modern instruments, the sample is scanned with a sharp tip mounted on a cantilever by means of a piezoelectric scanner. At the same time, the deflection of the cantilever is monitored through the reflection of a laser beam from its back. Initially, images were recorded in the contact mode. Thereby, the tip is brought into contact with the sample and scanned over the sample. More recently, one often prefers to excite the oscillation of the cantilever near its natural oscillation frequency. This approach results in a larger sensitivity to small variations of the interaction potential. In ultra-high vacuum, one often uses the non-contact mode, which explores the attractive part of the interaction potential. Due to the high quality factors of the cantilevers, frequency modulation (FM) mode is used to acquire the image. Thereby, one keeps the oscillation amplitude constant and always excites the cantilever at the resonance frequency. The image is generated by adjusting the distance to the sample such that the shift in the resonance frequency remains constant. In air and in solution, the intermitted-contact or tapping mode is preferred, whereby the tip approaches the sample in an intermitting fashion. Thereby, one uses the amplitude modulation (AM) mode, which keeps the amplitude of the oscillating cantilever constant.

In the past, the oscillation of the cantilevers was excited by an oscillating piezoelectric crystal. Particularly in liquids, the crystal not only excites the oscillation of the cantilever, but also generates sound waves in the liquid, which lead to a complicated response function. The current way to resolve this problem is photothermal excitation, whereby the cantilever is locally heated by a modulated laser beam. ${ }^{[10]}$ This excitation mode results in a close to ideal response of the cantilever, and leads to higher stability in image acquisition.

Further recurring problems in recording images in solution are the poor quality factors of the cantilevers, which are caused by the 


\section{Contact Mode}
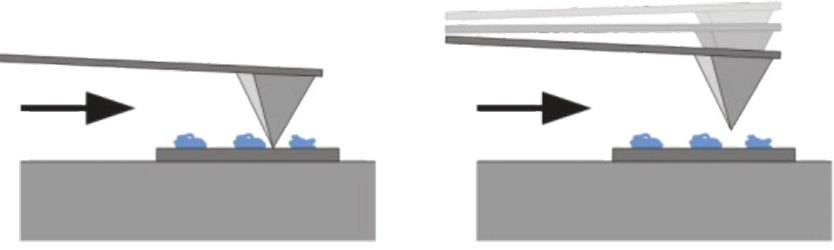

Fig. 1. Scheme of the atomic force microscope (AFM). The left cartoon depicts the contact imaging mode, and the right one the intermittentcontact mode.

substantial damping in liquids, which have much higher viscosities than air or vacuum. Modern micro-fabrication techniques now allow production of much smaller cantilevers than possible in the past. ${ }^{[11]}$ A smaller cantilever has a higher natural oscillation frequency and also experiences smaller damping. These two effects lead to much higher quality factors, which makes the cantilever more sensitive, and permits the generation of images in liquids even for low contrast samples. While normal AFM cantilevers have natural resonance frequencies in the $\mathrm{kHz}$ range, for modern ultra-small cantilevers this number increases to a few $100 \mathrm{kHz}$. Thereby, the quality factor in solution may increase by a factor 10 or more. This improvement leads to higher resolution in liquids and higher acquisition rates, opening even the possibility to record videos.

\section{Imaging of Single Polyelectrolytes}

Given the possibilities offered by these new AFM techniques, let us now demonstrate how individual polyelectrolyte molecules can be imaged in situ. All images shown here were recorded in aqueous solutions with the Cypher ES AFM (Oxford Instruments) with biolever-mini cantilevers (Olympus) with a resonance frequency in air of $130 \mathrm{kHz}$. The structures of the different polyelectrolytes used here are summarized in Fig. 2.<smiles>CC(C)c1cccc[nH+]1</smiles>

P2VP

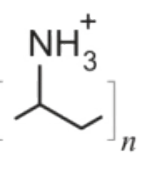

PVA

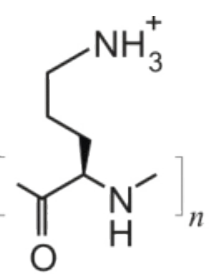

PLL

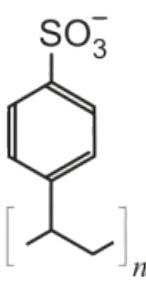

PSS
Fig. 2. Chemical structure of the polyelectrolytes used. Poly(2vinylpyridine) (P2VP), poly(vinylamine) (PVA), poly-L-lysine (PLL) and poly(styrene sulfonate) (PSS).

Let us start with images of poly(2-vinylpyridine) (P2VP). The samples used have a molecular mass of $160 \mathrm{~kg} / \mathrm{mol}$ and they were dissolved at a concentration of $0.1 \mathrm{mg} / \mathrm{L}$ in $10 \mathrm{mM} \mathrm{KCl}$ solution adjusted to $\mathrm{pH} 2.5$. These solution conditions are essential to obtain quality images of single molecules. At higher $\mathrm{pH}$ and at higher salt levels, the chains collapse, and it becomes impossible to resolve the individual chains. A small aliquot of the solution was placed on a freshly cleaved mica sheet and incubated for 1 min. The substrate was then rinsed with the same polymer-free electrolyte solution, and mounted in sealed AFM cell containing the same solution. Drying of the sample could be avoided. The $256 \times 256$ pixel images were recorded in AM-mode with a free oscillation amplitude between $1-10 \mathrm{~nm}$, which was determined by the GetReal method. ${ }^{[12]}$ The set-point was chosen to lie between $60-80 \%$ of the free oscillation amplitude.
Fig. 3 shows several images of P2VP adsorbed on mica recorded in the electrolyte solution. One observes well-resolved, mostly individual polymer chains. The chains have a height of about $0.4 \mathrm{~nm}$, and a cross-section of about $4 \mathrm{~nm}$. The latter number exceeds the actual cross section due to tip convolution artifacts. The adsorbed chains have all random coil conformations, but otherwise their structure is highly variable. Moreover, the polymer chains are firmly immobilized on the substrate. This aspect can be verified by acquiring an image of the same polyelectrolyte molecule at a later time and by demonstrating that this image is basically identical to the initial one. One may thus suspect that the chains did equilibrate laterally prior to the adsorption, but once the positively charged P2VP comes in contact with the negatively charged substrate, they adsorb firmly and irreversibly. One further observes brighter (higher) spots in the images at points where the chains re-cross themselves. Considering the substantial size of these spots, one may suspect that the chains coil up near these re-crossing points, and create pearl-like structures.
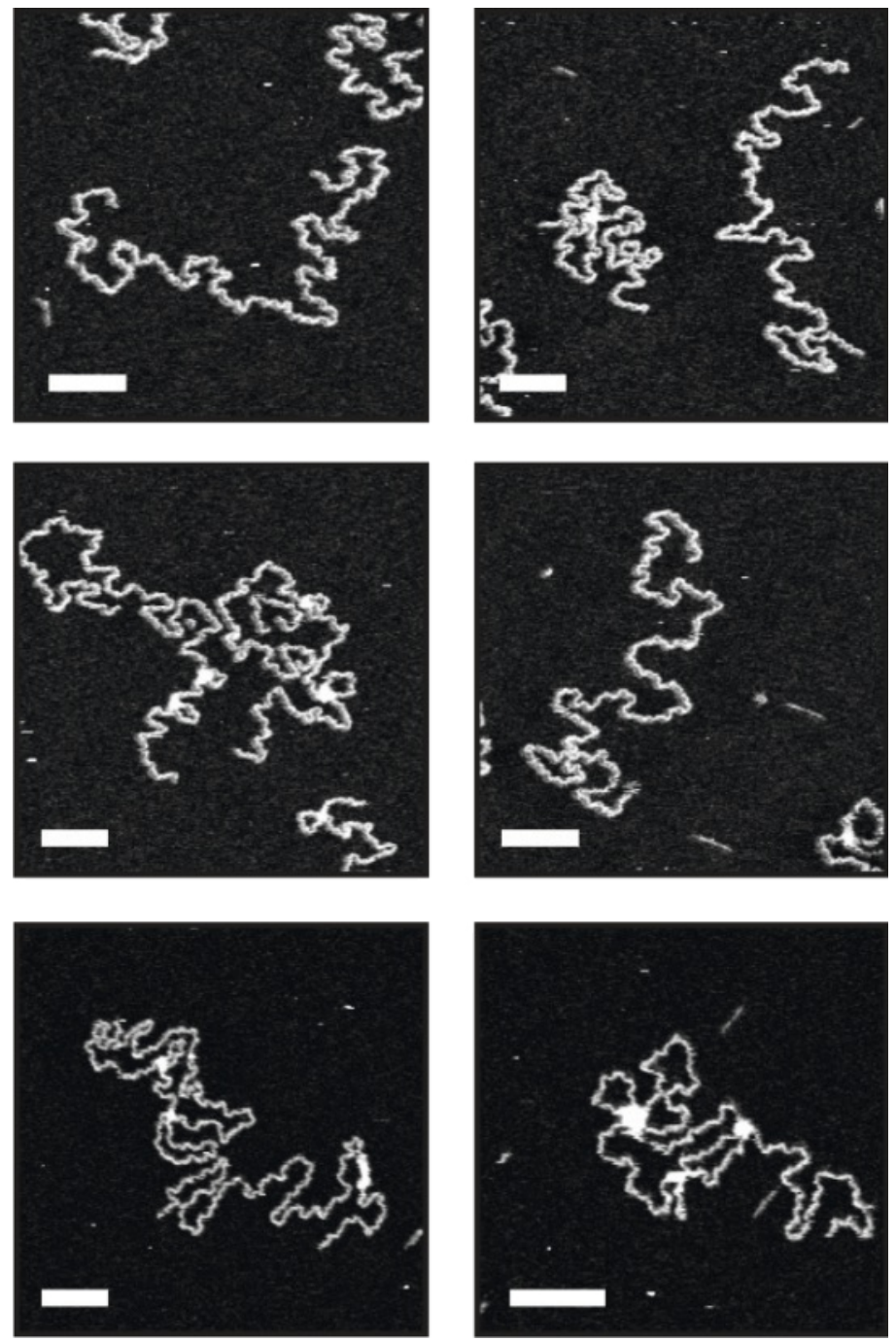

Fig. 3. Images of P2VP adsorbed on mica in $10 \mathrm{mM} \mathrm{KCl}$ solution of $\mathrm{pH}$ 2.50. The different images were recorded on various spots on the same sample. The scale bar is $20 \mathrm{~nm}$.

Fig. 4 shows images of other types of cationic polyelectrolytes recorded under similar conditions. The images of PVA are of comparable quality as for $\mathrm{P} 2 \mathrm{VP}$, but the chains show re-crossings more frequently. One also observes a larger number of globular pearl-like structures. Formation of pearl-necklace structures was evidenced for PVA by AFM imaging in air by some of us earlier. ${ }^{[13]}$ Similar pearl-necklace structures were also observed for 
other polyelectrolytes. ${ }^{[14]}$ In fact, the existence of such structures was predicted theoretically, provided the polyelectrolyte is highly charged and that the backbone is sufficiently hydrophobic. ${ }^{[15]}$ The images of PLL reveal that this polyelectrolyte leads to completely different structures upon adsorption. In this case, the polymer chain now adsorbs in straight conformations, but occasionally makes sharps turns of $60^{\circ}$. This structure suggests that PLL interacts with mica in a specific manner, and that the conformations of the adsorbed chains are templated by the hexagonal lattice structure of the principal cleavage plane of mica. The longer side chains of PLL probably provide the necessary flexibility, such that the positively charged amine groups can coordinate the negatively charged aluminum atoms at the mica surface. Similar template effects were also observed for adsorbed DNA on graphite.[16,17] These techniques can also be used to adsorb negatively charged PSS, see Fig. 4c. In this situation, the adsorbed chains are highly coiled, and in spite the frequent re-crossings, there seems to be little tendency of pearl formation.
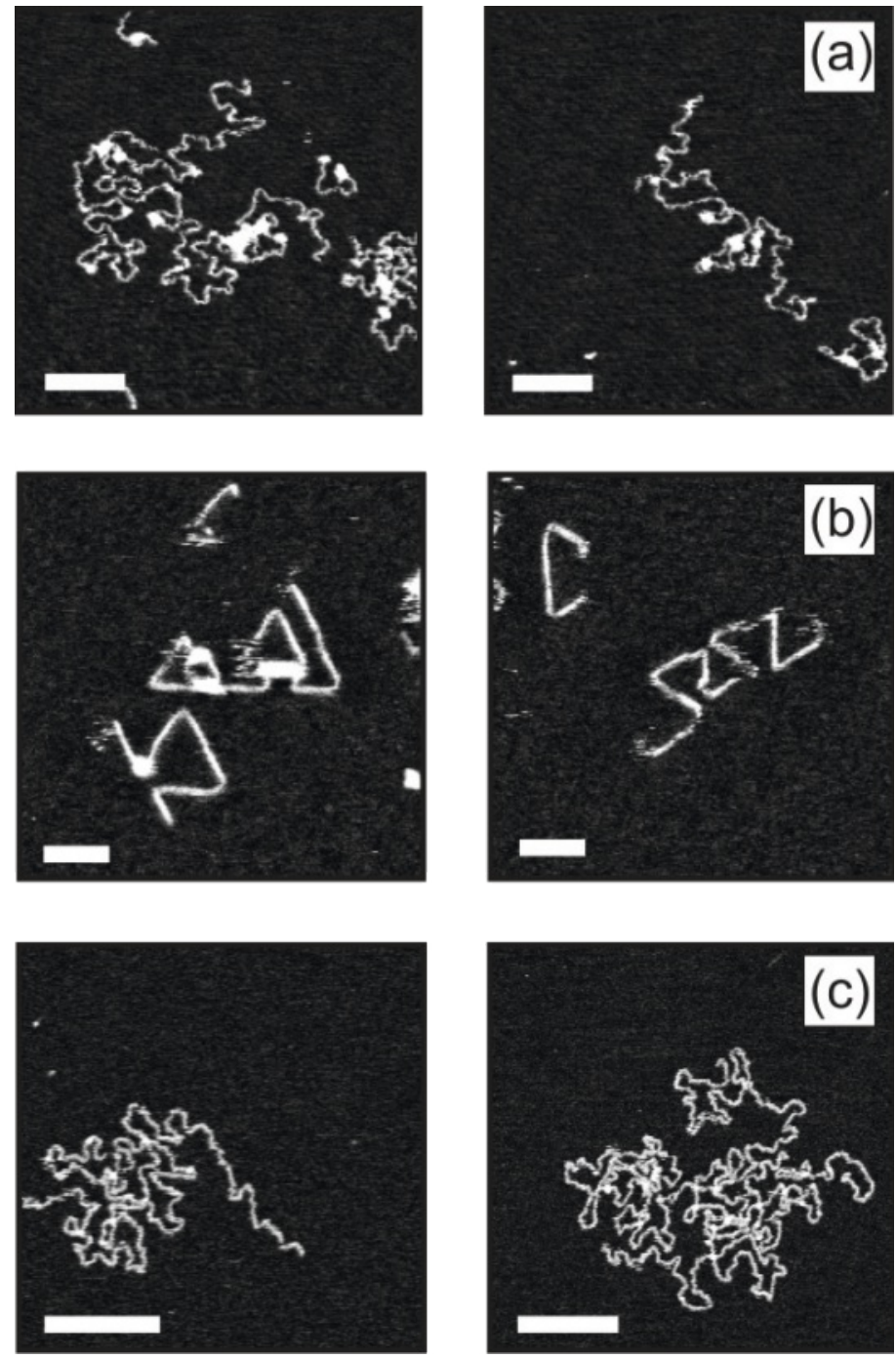

Fig. 4. Different polyelectrolytes adsorbed on mica imaged in electrolyte solutions. (a) PVA in $10 \mathrm{mM} \mathrm{KCl}$ solution of $\mathrm{pH}$ 2.50. (b) PLL in $10 \mathrm{mM}$ $\mathrm{KCl}$ solution of $\mathrm{pH}$ 4.0. (c) PSS in $10 \mathrm{mM} \mathrm{KCl}$ solution and $\mathrm{pH} 4.0$. The scale bar is $20 \mathrm{~nm}$.

\section{Determination of the Persistence Length}

The high quality images of the individual polyelectrolytes can also be analyzed quantitatively. As an example let us present the evaluation of the persistence length of the adsorbed polyelectrolytes with the worm-like chain (WLC) model.. ${ }^{[18]}$ One assumes that the chain has no internal structure and can be modeled as a flexible rod in a plane. The basic assumption of this model is that the directional correlation function decays exponentially. This function represents the average of the scalar product of two tangent unit vectors $\mathbf{n}$ that are evaluated at two positions separated by the contour length $s$ and is normally written as

$$
\langle\mathbf{n}(s) \cdot \mathbf{n}(0)\rangle=\exp \left(-\frac{s}{2 \ell}\right)
$$

where $l$ is the persistence length. Based on this assumption, one finds that the mean square average of the internal distance $\rho$ becomes

$$
\left\langle\rho^{2}(s)\right\rangle=4 \ell s-8 \ell^{2}\left[1-\exp \left(-\frac{s}{2 \ell}\right)\right]
$$

The limiting behavior of the above quantity

$$
\left\langle\rho^{2}(s)\right\rangle=\left\{\begin{array}{c}
s^{2} \text { for } s \ll \ell \\
4 \ell s \text { for } s \gg \ell
\end{array}\right.
$$

can be simply interpreted. For short distances, the chain can be approximated with a rigid rod, while for long distances it behaves as a Gaussian chain.

These quantities can be evaluated from the AFM images of the adsorbed polyelectrolytes. ${ }^{[19]}$ Here the procedure will be illustrated with the P2VP images shown in Fig. 2. The polymer backbone was digitized for 63 molecules, and from the respective planar coordinates the correlation function and the internal mean square displacement were calculated. The results are shown together with the best fits with Eqns. (1) and (2) in Fig. 5. The limiting laws given by Eqn. (3) are equally indicated in Fig. 5b. From the best fits one extracts a persistence length $l=2.3 \mathrm{~nm}$ from the correlation function and $l=2.1 \mathrm{~nm}$ from the internal distance. The good agreement between these two estimates demonstrates the consistency of the data analysis. The same type of analysis was also carried out for the PVA molecules shown in Fig. 3, where a somewhat smaller persistence length of $1.6 \mathrm{~nm}$ was found. ${ }^{[9]}$ Similar techniques were used earlier to determine the substantially larger persistence of DNA from AFM images obtained in the dry state. ${ }^{[20]}$

\section{Conclusion}

The aim of this short article was to illustrate the power of modern AFM techniques to image single adsorbed polyelectrolytes in aqueous solutions under in situ conditions. The key improvement is the use ultra-small AFM cantilevers, which lead to excellent resolution even for ambient conditions in liquids. This development permits to record high quality images of single polyelectrolyte molecules, and pinpoint important differences in the adsorbed conformations. Moreover, the images of adsorbed polyelectrolyte chains can be analyzed quantitatively. This type of analysis permits the persistence length of the adsorbed polyelectrolytes to be extracted.

\section{Acknowledgement}

This research was supported by the National Center of Competence in Research (NCCR) for Bio-Inspired Materials and University of Geneva. 

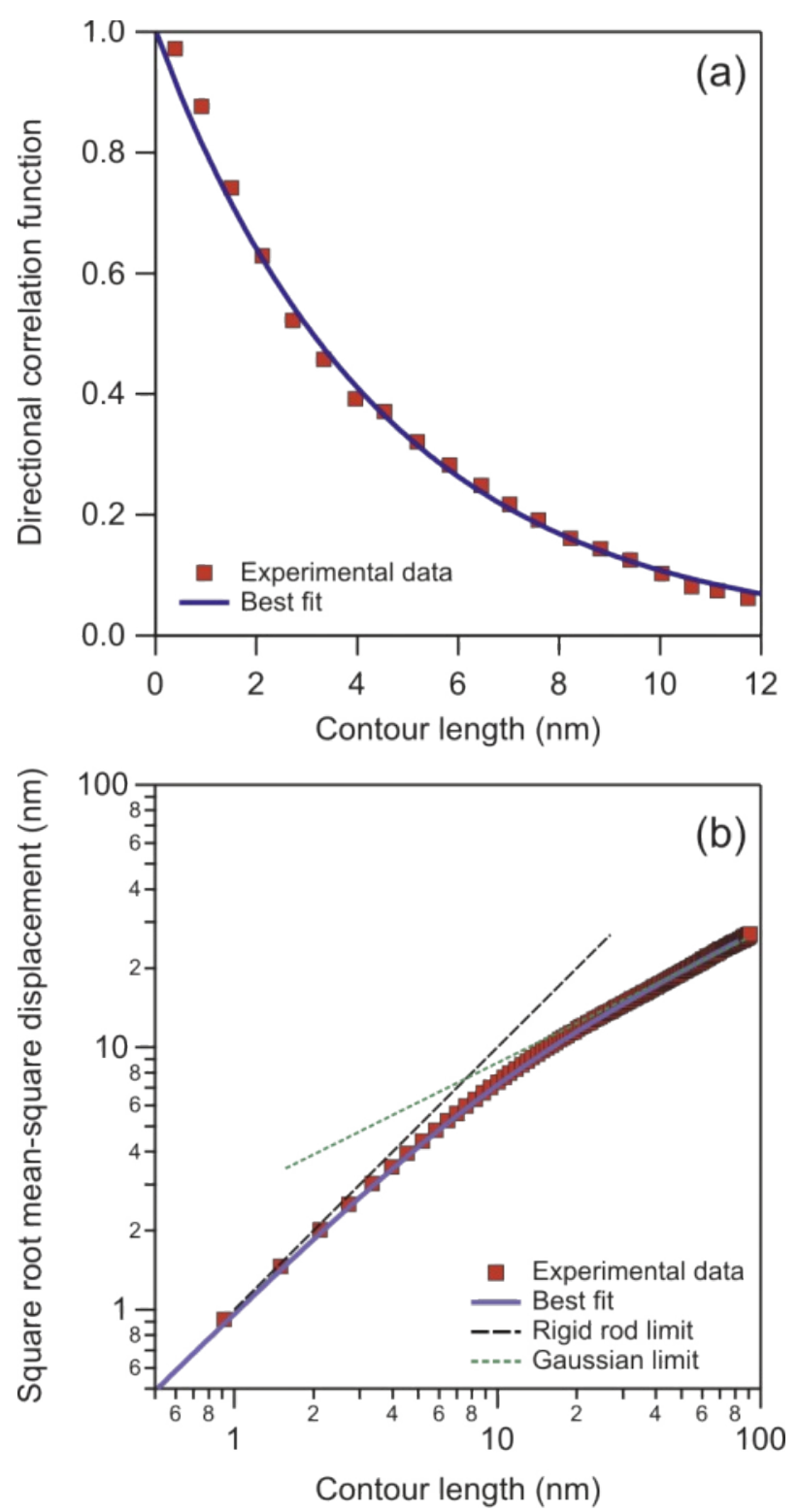

Fig. 5. Data obtained from quantitative image analysis of P2VP adsorbed on mica. (a) Exponential directional correlation function together with a scheme of the WLC model. (b) Corresponding internal mean square displacement. The latter subfigure also shows the limiting behavior of the rigid rod and a Gaussian chain. Both approaches lead to a persistence length of $2.2 \mathrm{~nm}$.
[1] M. G. L. Gustafsson, J. Microsc. 2000, 198, 82.

[2] M. Heilemann, S. van de Linde, M. Schuttpelz, R. Kasper, B. Seefeldt, A. Mukherjee, P. Tinnefeld, M. Sauer, Angew. Chem. Int. Ed. 2008, 47, 6172.

[3] G. Binnig, C. F. Quate, C. Gerber, Phys. Rev. Lett. 1986, 56, 930.

[4] M. Rief, M. Gautel, F. Oesterhelt, J. M. Fernandez, H. E. Gaub, Science 1997, 276, 1109.

[5] M. Rief, F. Oesterhelt, B. Heymann, H. E. Gaub, Science 1997, 275, 1295.

[6] T. Hugel, M. Grosholz, H. Clausen-Schaumann, A. Pfau, H. Gaub, M. Seitz, Macromolecules 2001, 34, 1039.

[7] M. Seitz, C. Friedsam, W. Jöstl, T. Hugel, H. E. Gaub, ChemPhysChem 2003, 4, 986.

[8] S. Minko, Y. Roiter, Curr. Opin. Colloid Interface Sci. 2005, 10, 9.

[9] S. Kozhuharov, M. Radiom, P. Maroni, M. Borkovec, Macromolecules 2018, 51,3632 .

[10] T. Fukuma, Rev. Sci. Instrum. 2009, 80, 023707.

[11] M. B. Viani, T. E. Schaffer, G. T. Paloczi, L. I. Pietrasanta, B. L. Smith, J. B. Thompson, M. Richter, M. Rief, H. E. Gaub, K. W. Plaxco, A. N. Cleland, H. G. Hansma, P. K. Hansma, Rev. Sci. Instrum. 1999, 70, 4300.

[12] M. J. Higgins, R. Proksch, J. E. Sader, M. Polcik, S. Mc Endoo, J. P. Cleveland, S. P. Jarvis, Rev. Sci. Instrum. 2006, 77, 013701.

[13] L. J. Kirwan, G. Papastavrou, M. Borkovec, S. H. Behrens, Nano Lett. 2004, 4, 149.

[14] A. Kiriy, G. Gorodyska, S. Minko, W. Jaeger, P. Stepanek, M. Stamm, J. Am. Chem. Soc. 2002, 124, 13454.

[15] A. V. Dobrynin, M. Rubinstein, S. P. Obukhov, Macromolecules 1996, 29, 2974.

[16] A. M. O. Brett, A. M. C. Paquim, Bioelectrochemistry 2005, 66, 117.

[17] J. Adamcik, S. Tobenas, G. Di Santo, D. Klinov, G. Dietler, Langmuir 2009, 25, 3159.

[18] A. Y. Grosberg, A. R. Khokhlov, 'Statistical Physics of Macromolecules', American Institute of Physics, Woodbury, 1994.

[19] A. Mikhaylov, S. Sekatskii, G. Dietler, J. Adv. Microscopy Res. 2013, 8, 241.

[20] F. Valle, M. Favre, P. de los Rios, A. Rosa, G. Dietler, Phys. Rev. Lett. 2005, 95, 158105 